\title{
Modification of the Projection from Anterior-Posterior to Posterior-Anterior for Abdominal Radiographic Examinations
}

\author{
Ghassan Abdullah Almaziad1,2,3, Abdullah Al Shahrani1,2,3, Oinam Gokulchandra Singh 2,3,4, \\ Ali Asghar Ayaz1, 2, 3 and Fatema Ibrahim Abudiab and Sulaiman O. Balogun1,2,3 \\ ${ }^{1}$ Medical Imaging Department, King Abdulaziz Medical City (KAMC), \\ Riyadh, Kingdom of Saudi Arabia \\ ${ }^{2}$ King Abdullah International Medical Research Centre (KAIMRC), Riyadh, \\ Kingdom of Saudi Arabia \\ ${ }^{3}$ National Guard Health Affairs (NGHA), Riyadh, Kingdom of Saudi Arabia \\ ${ }^{4}$ King Saud Bin Abdulaziz University for Health Sciences (KSAU-HS), Riyadh, \\ Kingdom of Saudi Arabia
}

\section{ABSTRACT}

The technological development improvement in healthcare, specifically in medical imaging, resulted in an increase in the number of examinations performed in diagnostic medical imaging. These examinations involve the use of ionizing radiation in both adult and pediatric patients. The aim of the study is to provide the foundation for the modification of the current practice of using Anterior-posterior (AP) projection in favor of Posterior-Anterior (PA) projection. The modification will facilitate adherence to the "as low as reasonably achievable" (ALARA) principle. This study was conducted at the Main X-ray of the Radiology Department, Ministry of National Guard-Health Affairs (MNGHA), King Abdulaziz Medical City (KAMC), Riyadh, Saudi Arabia. A cross-sectional study, with an experimental research study, were performed and measured in different phases. We included 110 patients (57 males, and 53 females) who required an abdominal radiographic examination. The majority (63) were exposed to the AP and 47 to the PA orientation. A nonparametric Mann Whitney U test was used to analyze the data. A total of 110 patients participated in this study. Statistical significance $(\mathrm{P}<0.001)$ was observed in the overweight Body mass index (BMI) category, with a mean Dose area product (DAP) of $11.8 \mathrm{dGycm}^{2}$ and $6.8 \mathrm{dGycm}^{2}$ for the AP and PA projections. In the overweight BMI category, the Entrance Skin Dose (ESD) was $1.51 \mathrm{mGy}$ and $0.83 \mathrm{mGy}$ for the AP and PA projections, respectively, with a statistically significant $58.11 \%$ difference $(\mathrm{P}<0.001)$. Similarly, in the obese BMI category, the ESD was $2.35 \mathrm{mGy}$ and $1.6 \mathrm{mGy}$ for the AP and PA projections, indicating a statistically significant $37.97 \%$ difference $(\mathrm{P}<0.046)$. The study provided evidence that the PA projection is an easily implementable method to reduce the dose, with no loss of diagnosis for the patient and no additional cost to the department.

KEY WORDS: RADIOGRAPHIC EXAMINATION, ANTERIOR-POSTERIOR, POSTERIOR-ANTERIOR, ENTRANCE SKIN DOSE, DOSE AREA PRODUCT, BODY MASS INDEX.

\section{INTRODUCTION}

The continuous advancement in technology has impacted

Biosc Biotech Res Comm P-ISSN: 0974-6455 E-ISSN: 2321-4007

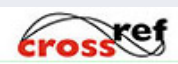

Identifiers and Pagination

Year: 2021 Vol: 14 No (5) Special Issue

Pages: 258-264

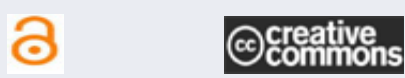

Article Information

Received: $18^{\text {th }}$ Mar 2021

This is an open access article under Creative

Commons License Attribn 4.0 Intl (CC-BY).

DOI: http://dx.doi.org/10.21786/bbrc/14.5/47 the science field specifically the medical field; thereby, improving the delivery of healthcare. Radiology is a branch of medicine where technology has revolutionized and transformed patient care delivery. Many diseases are frequently first detected through diagnostic medical imaging. However, the technological advancement witnessed in medical imaging has led to an increased number of examinations that involve the use of ionizing radiation in both adult and pediatric patients (Furlow, 2011). Radiography constitutes the highest number of examinations performed for patients of all 
age groups, when compared to other imaging modalities (Don et al., 2013).

According to Furlow (2011), "the frequency of diagnostic radiologic examination for patients of all ages in the United States has increased by 10-fold since 1950" (p. 421). Strict adherence to one of the main principles of radiation protection policies, "as low as reasonably achievable" (ALARA) and protecting a vulnerable group of patients appropriately from unnecessary radiation exposure should be paramount while delivering excellent patient care services with diagnostic ionizing radiation (Brennan \&t Madigan, 2000; Don, 2011; Furlow, 2011). The Ministry of National Guard-Health Affairs' Administrative Policy and Procedures (APP 1430-43), stated that "As Low as Reasonably Achievable (ALARA) refers to a radiation safety principle for minimizing radiation doses by employing all reasonable methods. ALARA is not only a sound safety principle but is a regulatory requirement for all radiation safety programs" (4.1).

Due to the undesirable effects, which unnecessary radiation exposure from diagnostic ionizing radiation procedures could cause, it is important to explore all available avenues and strategies to reduce the patient radiation dose? One important strategy to reduce and prevent an excessive radiation dose a patient is exposed to, is the modification of patient's position from the anterior posterior (AP) to a posterior anterior (PA) projection (Brennan \&t Madigan, 2000).

Radiographs of the lower and upper extremities, abdomen, and chest are a component of plain film imaging, which are categorized under projection radiography. These radiographs constitute the majority of the imaging examinations performed in the diagnostic imaging department, of most hospitals for both adult and pediatric patients (Don, 2011; Moore, 2015). The sensitivity of the human organs and tissues varies, as some are more sensitive to radiation than others (Furlow, 2011; Moore 2015). The majority of the sensitive tissues and organs, such as the colon, breasts, liver, intestines, and stomach, are located in the anterior portion of the body (Furlow, 2011; Moore 2015). As stated by Moore (2011), when projection radiography examinations of the chest, pelvis, abdomen, and spine are performed in the AP projection, patients are exposed to a high "surface dose" of ionizing radiation. Radiation exposure to organs and tissues located anteriorly would be significantly reduced by changing the patient orientation from AP to PA by the performing technologist (Furlow, 2011; Moore 2015).

In an abdominal examination, "AP projections give rise to higher effective doses than do PA projection because the majority of the irradiated radiosensitive organs in the abdomen, such as the stomach, the colon and the liver, lie closer to the entrance surface for AP views compared with PA views" (Marshall et al. 1994, p. 482). Performing an abdominal x-ray examination in the PA position, as a radiation dose reduction strategy, has been supported in literature highlighting that significant dose reductions can be achieved with a projection modification from
AP to PA (Marshall et al. 1994). In the study carried out by Ghearr and Brennan (1998), they discovered that an effective dose and the entrance surface dose to the patient was reduced when the PA projection of the abdomen was used, with no changes noted in the overall image quality in the two methods. Changing the patient's position from a supine AP projection to a prone PA projection would play a significant role in reducing the patient's radiation exposure because the abdominal "soft" tissues are compressed in the prone position, and lower exposure factors will be required to obtain the image (Moore 2015).

The aim of the study was to provide empirical evidence that will help to create awareness among decision makers, stakeholders, and change the policy and procedures, primarily in the MNGHA-KAMC, and generally in Saudi Arabia to accommodate the new protocol by utilizing the benefits of using a PA projection as compared to an AP projection when conducting a abdominal radiographic examination. This will provide the foundation to support the modification of the current practice of using an AP-projection in favor of a PA-projection, keeping in line with the adherence to the "as low as reasonably achievable" (ALARA) principle. The goal of reducing and avoiding unnecessary ionizing radiation dose to patients should be highly prioritized in diagnostic medical imaging. Except in some condition of the patients that requires performing AP projections such as: 1- Critical post procedure patients. 2- Intubated on suction patients. 3- Portable case or bedside abdominal exams. 4- Pregnant patient, which consider as a limitations for the current study, modification of the projection from AP to PA for abdominal radiographic examinations will be a step in the right direction.

Research Question: The research question sought to determine if there are any differences in the ESD and DAP values recorded during an abdomen radiography exam under the AP-positioning or the PA-positioning.

\section{MATERIAL AND METHODS}

This study was conducted at the Main X-ray of the Radiology Department, Ministry of National Guard Health Affairs (MNGHA), King Abdul-Aziz Medical City (KAMC), Riyadh, Saudi Arabia. A cross-sectional study, with an experimental study, were performed and measured in different phases. We included 110 patients who required an abdominal radiographic examination, and the majority (63) were exposed to the AP orientation and 47 patients were exposed to the PA orientation. In terms of gender, there were 57 males and 53 females. A comparison of the AP and PA abdominal x-ray procedures were done. For this purpose, one digital radiography unit in the Medical Imaging Department was chosen as the setting. During the initial investigation, a test was performed on an anthropomorphic phantom, as shown in the Image 1 (RS-111T, manufactured by Radiology support Devices INC, RDS). It is a rugged sectional representation of an average male, $175 \mathrm{~cm}$ tall, weighing $74 \mathrm{~kg}$, which provides a comprehensive 
platform for the evaluation of imaging systems and imaging techniques in realistic conditions.

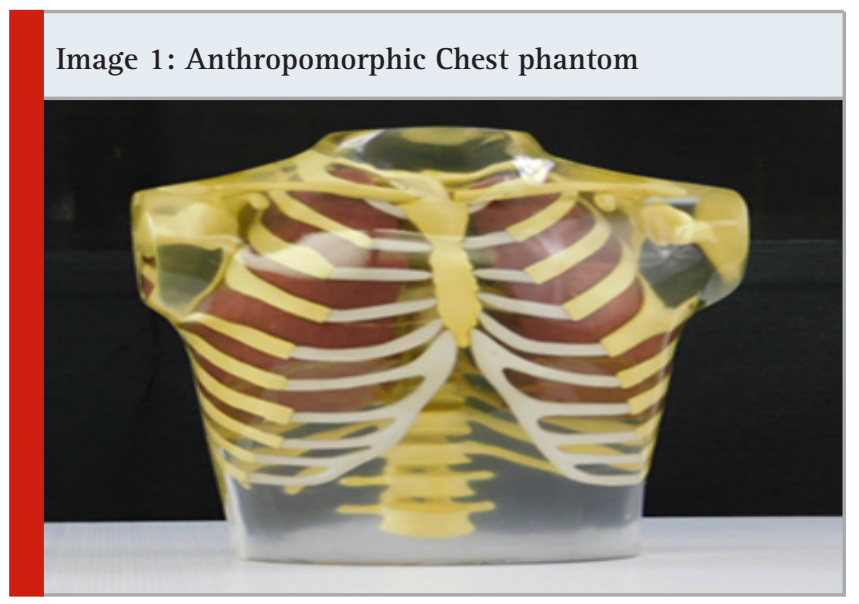

Table 1. Basic Exposure Characteristics of Phantom Study

\begin{tabular}{|l|c|}
\hline $\mathrm{kVp}$ & 100 \\
\hline $\mathrm{SID}$ & 100 \\
\hline Grid & $100 \mathrm{~cm}$ focused grid \\
\hline Collimation & $33 \mathrm{~cm} \times 26 \mathrm{~cm}$ \\
\hline
\end{tabular}

Image 2: Raysafe X2 Dosimeter set

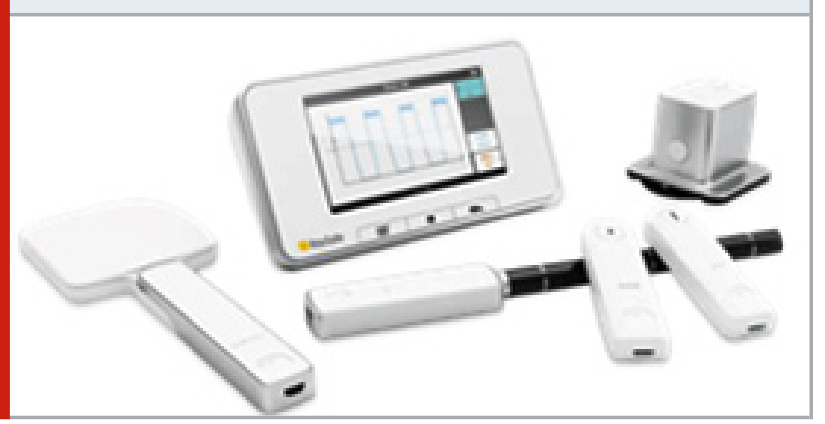

The phantom was scanned under both the AP and PA projections. In the next step, in a duration of 6 months, from May 2019 to October 2019, patients requiring an abdominal $\mathrm{x}$-rays were examined into an AP and PA projections depending on patient condition. A GE Revolution XR-D digital radiography machine, with a digital detector was used to scan both groups with the automatic exposure control (AEC) system turned on. Basic exposure characteristics for the phantom measurement are shown in Table 1 . All the abdominal examinations were performed at a nominal tube voltage of $80 \mathrm{kVp}$. The grid used in the study had a 12:1 grid ratio with 78 lines $/ \mathrm{cm}$ and a focusing distance of $100 \mathrm{~cm}$. Before conducting the study, a qualified medical physicist performed a complete quality control test of the machine using a calibrated Raysafe X2 dosimeter (Image 2). It is used for radiography and fluoroscopy measurements with or without a phantom between the sensor and the X-ray source. The baseline values for the entrance skin dose were measured using a $20 \mathrm{~cm}$ thick ACR Radio/Fluoro patient phantom with $80 \mathrm{kVp}$ exposures at different ranges of $m A s$ and source to phantom distances. The dose area product (DAP) measurements were taken from the built-in DAP meter in the tube housing.

Table 2. Scoring system for Image quality

\begin{tabular}{|l|l|}
\hline Score & Description \\
\hline Inadequate & $\begin{array}{l}\text { The anatomical feature is not visualized } \\
\text { or is detectable but the details are } \\
\text { not fully reproduced }\end{array}$ \\
\hline Adequate & $\begin{array}{l}\text { The details of anatomical features are } \\
\text { visible but not necessarily clearly defined }\end{array}$ \\
\hline Perfect & The anatomical details are clearly defined \\
\hline
\end{tabular}

Table 3. Image Quality assessment criteria

\begin{tabular}{|l|l|}
\hline & Criteria \\
\hline 1 & $\begin{array}{l}\text { Complete Visualization of the } \\
\text { anatomy on the image (kidney outline, } \\
\text { liver, spine pelvic bones and liver }\end{array}$ \\
\hline 2 & Sharpness of the image \\
\hline 3 & Visualization of important area and organ \\
\hline 5 & $\begin{array}{l}\text { Detection of opacified calcification } \\
\text { No blurring and good visualization of } \\
\text { bowel gases and air fluid levels }\end{array}$ \\
\hline
\end{tabular}

Table 4. Exposure and output in AP projection of the Phantom measurement

\begin{tabular}{|l|c|c|c|}
\hline Exposure & $\mathrm{mAs}$ & $\begin{array}{c}\text { Dose } \\
\mathrm{mGy}\end{array}$ & $\begin{array}{c}\text { DAP } \\
\mathrm{dGycm}^{2}\end{array}$ \\
\hline 1 & 4.71 & 4.66 & 18.27 \\
\hline 2 & 4.68 & 4.63 & 18.15 \\
\hline 3 & 4.72 & 4.67 & 18.31 \\
\hline Mean & 4.70 & 4.65 & 18.24 \\
\hline
\end{tabular}

Table 5. Exposure and output in PA projection of the Phantom measurement

\begin{tabular}{|l|c|c|c|}
\hline Exposure & $\mathrm{mAs}$ & $\begin{array}{c}\text { Dose } \\
\mathrm{mGy}\end{array}$ & $\begin{array}{c}\mathrm{DAP} \\
\mathrm{dGycm}^{2}\end{array}$ \\
\hline 1 & 4.34 & 4.30 & 16.84 \\
\hline 2 & 4.35 & 4.31 & 16.88 \\
\hline 3 & 4.34 & 4.29 & 16.82 \\
\hline Mean & 4.34 & 4.30 & 16.85 \\
\hline
\end{tabular}

Phantom Measurements: In the first phase of the study, we used chest phantom due to unavailability of Abdomen phantom at NGHA. The Phantom used in the project composed of chest and upper abdomen, and our main area of interest is being covered on this 
phantom. Measurements were carried out on a human body phantom for abdominal procedure. For all the exposures performed, parameters such as $\mathrm{kVp}$, SID, grid type and collimation were kept constant while changing the orientation of the phantom from AP to PA. Automatic exposure control (AEC) was used with central chamber turned on, the phantom was imaged 4 times in each orientation. Dose area product (DAP) values were recorded for each exposure along with entrance phantom dose measured by an external calibrated dosimeter placed on top of the phantom. The exposure mode was taken on Automatic Exposure Control AEC, so the exposure parameter mAs was set up automatically by the machine itself with respect to orientation of phantom whether AP or PA projection. In fact, due to the difference of structures in the anterior and posterior surface in composition, density and attenuation, different readings among AP and PA projections were recorded, although compressibility of phantom abdomen was not achievable.

Figure 1: Comparison of Dose area product (DAP) for AP and PA projection in the patient study

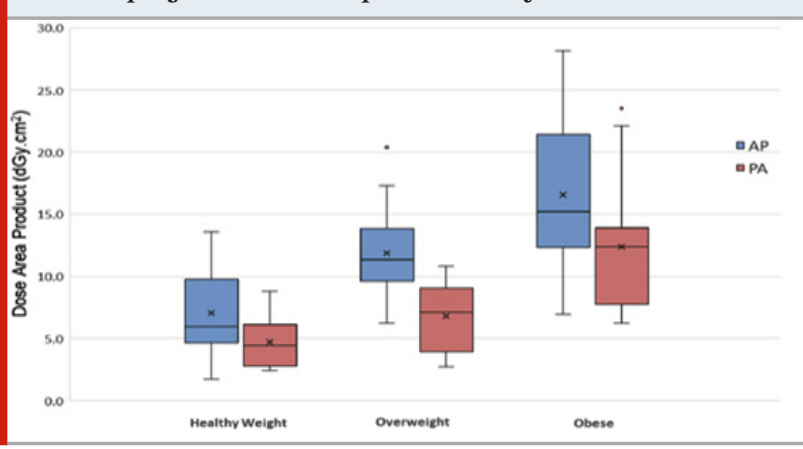

Figure 2: Comparison of the Entrance Skin Dose (ESD) for $\mathrm{AP}$ and PA projections

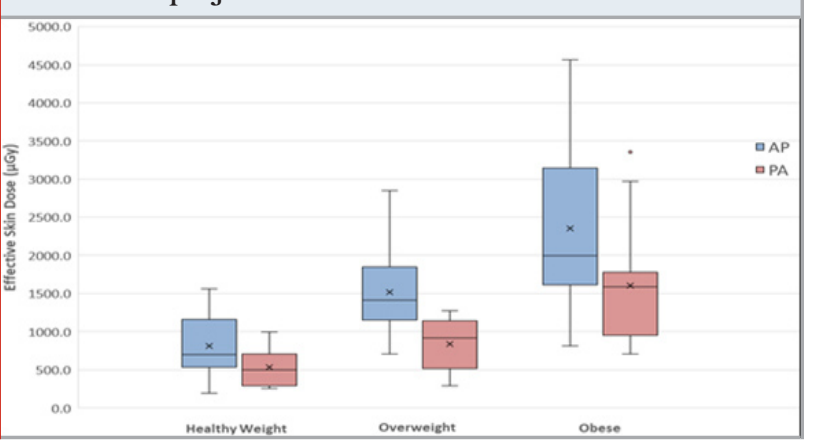

Patient Measurements: The second phase of the study comprised of the dose measurement with patients requiring an abdominal radiography examination. The patients were examined with an AP or PA orientation depending on patient condition over 6 months. Prior to their examination, the patient's height and weight were recorded and the BMI calculated. The source to skin distance (SSD) from the center of the field was measured and recorded. After the exposure, the mAs used by the system and the displayed dose area product values were also noted. The values of SSD and mAs were later used to calculate the entrance skin dose for each patient individually.

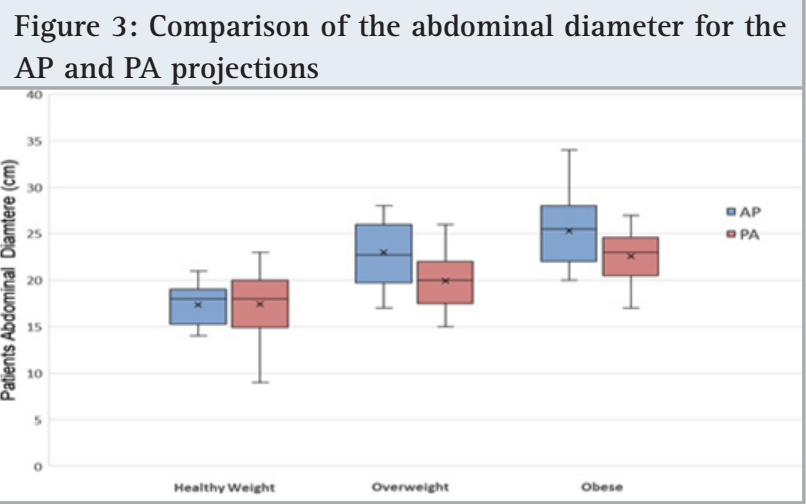

Table 6. Demographic characteristics $(n=110)$

\begin{tabular}{|l|c|}
\hline Variable & Number ct percentage \\
\hline Gender & \\
\hline Male & $57(51.8 \%)$ \\
\hline Female & $53(48.2 \%)$ \\
\hline Age, mean \pm SD & $58.74 \pm 18.93$ \\
\hline Body mass Index kg/m2 & $26(23.6 \%)$ \\
\hline Healthy & $38(34.5 \%)$ \\
\hline Overweight & $46(41.8 \%)$ \\
\hline Obese & \\
\hline
\end{tabular}

The approval of Institutional Research Board of King Abdullah International Medical Research Center (KAIMRC) was obtained prior to conducting the study. All the patients included in this study were informed about the purpose of this experiment and written consent was obtained before the examination.

Image Quality Assessment: Three experienced consultant radiologists working at King Abdul-Aziz Medical City, Riyadh, Saudi Arabia evaluated the radiographic images obtained in both AP and PA orientations. The radiologists reviewed and assessed the abdominal images of 80 patients, which were displayed in a randomized manner, with them not knowing the patient orientation through examination. During the image assessment, the radiologists were not allowed to do any image adjustments or magnification which could indicate an AP or PA projection of the image. They used a 3-point scale, according to the specific criteria for an abdominal projection, as issued in the European Guidelines on Quality Criteria for Diagnostic Radiographic Images (Doktor, Klaus et al). The ratings of the 3-point scale was: score 1 - inadequate, 2 - adequate and 3 - perfect (Table 2). Subsequently, five different standards were used to rate the quality of the images, based on complete visualization of the anatomy, image sharpness, important organs/area clearly visible and detection of opacified calcifications (Table 3). The criteria for the assessment was modified and adapted according to the protocol of KAMC, Riyadh, Saudi Arabia. 


\begin{tabular}{|c|c|c|c|c|c|}
\hline BMI & Sample Size & Patient Dose & Projection & Mean & P-value \\
\hline \multirow[t]{4}{*}{ Healthy } & \multirow{4}{*}{$\begin{array}{c}\text { Total }=26 \\
\mathrm{AP}=13 \\
\mathrm{PA}=13\end{array}$} & ESD (mGy) & $\mathrm{AP}$ & 0.81 & 0.045 \\
\hline & & \multirow{3}{*}{ DAP (dGy.cm2) } & PA & 0.53 & \\
\hline & & & $\mathrm{AP}$ & 7.00 & 0.0403 \\
\hline & & & PA & 4.70 & \\
\hline \multirow[t]{4}{*}{ Overweight } & \multirow[t]{4}{*}{$\begin{array}{c}\text { Total }=38 \\
\mathrm{AP}=23 \\
\mathrm{PA}=15\end{array}$} & \multirow[b]{2}{*}{ ESD (mGy) } & & & \\
\hline & & & $\begin{array}{l}\text { AP } \\
\text { PA }\end{array}$ & $\begin{array}{l}1.51 \\
0.83\end{array}$ & 0.001 \\
\hline & & \multirow[t]{2}{*}{ DAP (dGy.cm2) } & $\mathrm{AP}$ & 11.80 & 0.001 \\
\hline & & & PA & 6.80 & \\
\hline \multirow[t]{4}{*}{ Obese } & \multirow[t]{4}{*}{$\begin{array}{c}\text { Total }=46 \\
\mathrm{AP}=27 \\
\mathrm{PA}=19\end{array}$} & \multirow[t]{2}{*}{ ESD (mGy) } & AP & 2.35 & 0.046 \\
\hline & & & $\mathrm{PA}$ & 1.60 & \\
\hline & & \multirow[t]{2}{*}{ DAP (dGy.cm²) } & $\mathrm{AP}$ & 16.60 & 0.088 \\
\hline & & & PA & 12.40 & \\
\hline
\end{tabular}

Figure 4: Average scores of AP and PA images according to the radiologist

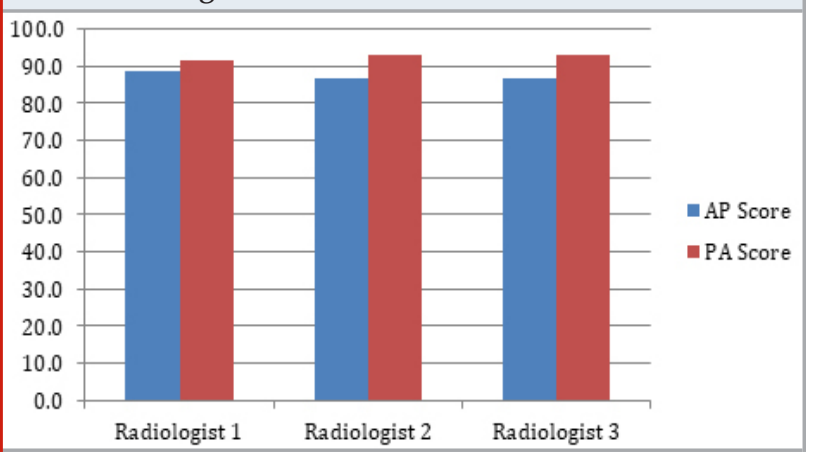

Figure 6: An AP projection of abdomen

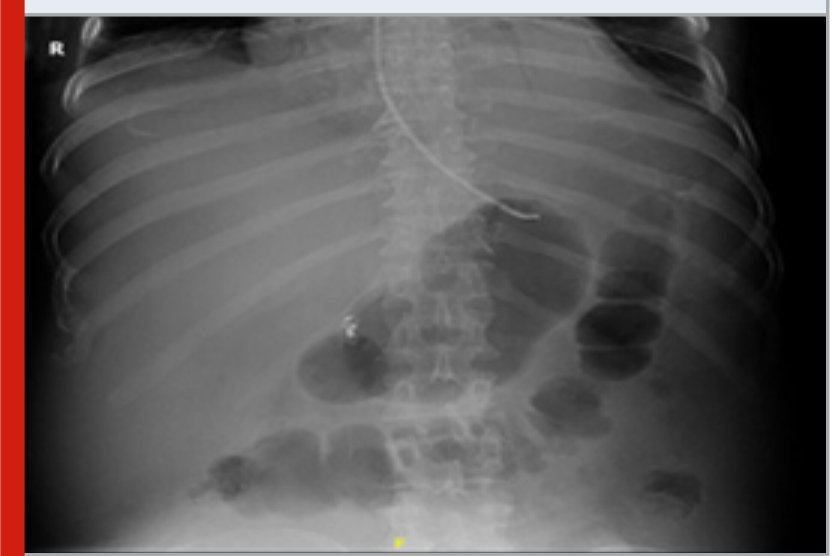

Data Management and Statistical Analysis: The data analysis was performed primarily by a medical physicist. The data was collected from May 2019 to October 2019 for 6 months period, entered in Microsoft Excel, and
Figure 5: Average scores of AP and PA images according to the image quality assessment criteria

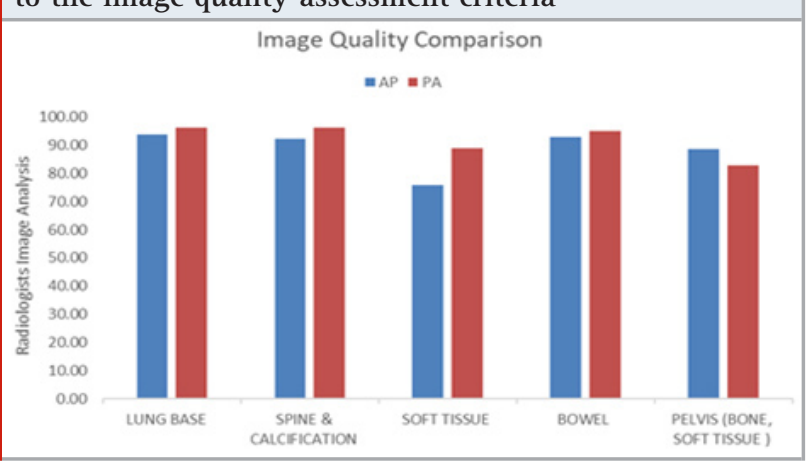

Figure 7: A PA projection of the abdomen

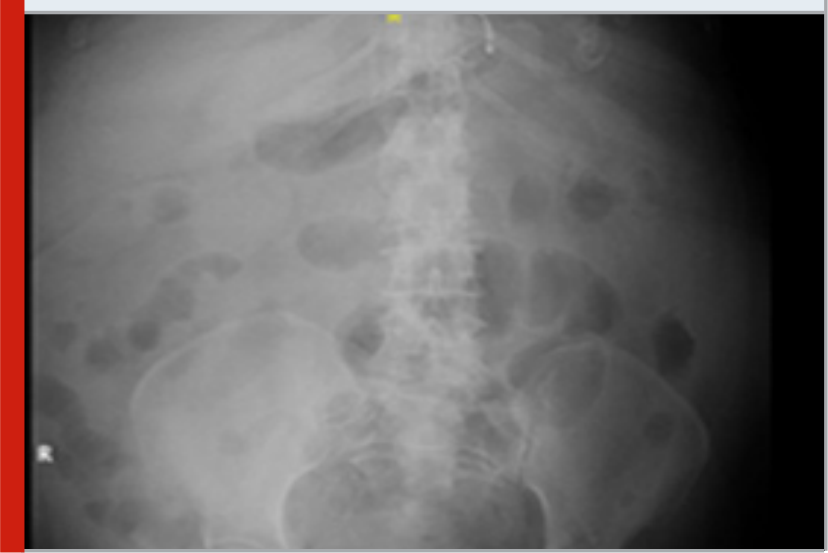

transferred to SPSS version 23 for statistical analysis. A nonparametric Mann Whitney U test was used. The results were based on the ESD in relation to the DAP of the abdominal x-ray examination for both the AP and 
PA positions. The significance cut-off point of $\mathrm{P}<0.05$ was used for the study. Finally, the findings are presented with relative difference and standard mean error and illustrated in graphs (Figures 1-3 and Tables 4-7).

\section{RESULTS}

The Phantom Study: Prior to the study, the measurements of an anthropomorphic phantom (RS-111T, manufactured by Radiology support Devices INC, RDS) were obtained. It is a rugged sectional representation of an average male, $175 \mathrm{~cm}$ tall, weighing $74 \mathrm{~kg}$. For all the measurements, parameters such as the $\mathrm{kVp}$, SID, grid type and collimation were kept constant. Based on the results, we concluded that by changing the orientation from AP to PA, we can decrease the patient dose, although it was not highly significant. This is mainly due to the nature of the phantom where abdominal compression, when oriented in the prone setup, cannot be achieved. The calibration of the machine, such as the built-in DAP was tested prior to study. The phantom was imaged 6 times, three times in the AP and then three times in the PA projection with AEC active in both projections (Tables 4 and 5). A mean ESD of $4.65 \mathrm{mGy}$ and $4.30 \mathrm{mGy}$ was measured for the $\mathrm{AP}$ and PA projections, and the mean DAP was 18.24 dGycm2 and 16.85 dGycm2 for AP and PA projection respectively. The organ dose, which will be much lower in the prone setup than the supine orientation, must be taken into consideration.

Patient Study: In the second phase of the study, we included 110 patients who had an abdominal radiographic examination, of which 63 were positioned in the AP and 47 in the PA orientation, and 57 were male with 53 females. The data was categorized into three groups based on the body mass index (BMI) as healthy, overweight, and obese. The choice of AP or PA depended on the condition of the patient. A GE machine with an X-ray beam energy of 80 KVP and 100 SID was used throughout the study. The demographic information is displayed in Table 6. All the data related to DAP, ESD and the patient's abdominal diameters are summarized in Table 7.

The DAP for the AP and PA projections of all the BMI categories are summarized in Figure 1 and Table 7. For the overweight BMI category, the mean DAP of 11.8 dGycm 2 and 6.8 dGycm 2 were recorded for the AP and PA projections respectively; hence, statistically significant $(\mathrm{P}<0.001)$, representing a difference of 53.76\%. Comparing the ESD for the AP and PA projections (Figure 2, and Table 7), the mean ESD was $813.7 \mu$ Gy and 531.7 $\mu \mathrm{Gy}$ for the AP and PA projections in the healthy BMI category, and statistically significant $(\mathrm{P}<0.045)$, though a $41.9 \%$ difference was recorded. However, in the overweight BMI category, an ESD of $1518.2 \mu \mathrm{Gy}$ and $833.1 \mu \mathrm{Gy}$ for the AP and PA projections, was statistically significant $(\mathrm{P}<0.001)$ with a difference of $58.27 \%$. For the obese BMI category, an ESD of $2350.2 \mu \mathrm{Gy}$ and $1607 \mu \mathrm{Gy}$ for the AP and PA projection was recorded, indicating a statistically significant difference of $37.56 \%(\mathrm{P}<0.046)$. For comparison analysis of the abdominal diameter for the AP and PA projection is illustrated in below Figure 3 whereby maximum value came from the obese category followed by overweight and healthy weight category.

Demographic Characteristics: From Table 7, 51.8\% were male and $48.2 \%$ female. The majority (63) were done with the AP projection, and 47 were performed in the PA projection. The mean age was 58.74 years. Based on the BMI kg/m2, the patients were categorized into three groups; healthy, overweight and obese.

Image quality: In the current study, the overall score of the evaluation of the three radiologists of the image criteria for the AP and PA projections, were slightly higher with the PA abdominal projection. Examining the criteria, the sharpness and the visual detection of opacified calcification was better in the PA position. From Figure 5 , the visualization of areas such as the spine, bowel and abdominal organs, was clearer compared with the anatomical structures, important for the initial patient assessment. It was concluded that fat lines, abnormal air fluid levels, and obstructed area were more visible in the PA than the AP projection. It should be noted that in some patients, the superimposition of the sacral bones over the pelvic structures, was the only criterion visualized better in the AP projection. To conclude the assessment of the image criteria, all three radiologists agreed that it was better using a PA projection for an abdominal examination, as this would not result in the loss of diagnostic information in the resultant image. All the images received a combined score of the criteria from a minimum point scale of 5 to a maximum of 15 points. The average score of all the AP and PA images of all the radiologists were calculated (Figure 4 \&t 5) and was $88 \%$ and $92 \%$ for the AP and PA projections respectively. An example of each radiographic projection is shown in Figures 6 and 7.

\section{DISCUSSION}

The main aim of the study was to compare the AP and PA projections of abdominal x-ray among 110 patients, both males and females, different sizes and different clinical conditions. The comparison was based on the measurement of different parameters when changing the technique from supine to prone position. Anatomy of internal abdominal organs (Soft tissue, bones, gas, fat lines and opacifications), patient radiation dose and image quality were measured in the current study. All patients were positioned for AP and PA in the same protocol followed in the department. We found that performing abdomen PA projection leads to overall reduction of DAP and effective dose significantly. Apparently, this significant dose reduction in the PA projection noticed more in patients with an obese and overweight BMI.

There was a significant difference in the soft tissue parameter, in the comparison between AP and PA projections, showed that PA was better. This was because abdominal thickness was compressed in prone position, which allows for more enhancement, decrease in scatter radiation and production of better quality. 
While AP projection is slightly better in pelvic bone anatomy due to two reasons: -

a- In PA position, the pelvic bones are away from detector no more in contact, which cause magnification and lead to slight image quality loss.

b- In addition, the prone position will cause in some patients overlapping of pelvic bones with intra pelvic structures obscuring some anatomy and decreasing image quality.

For all other parameters; the sharpness and visibility of lung bases, spine and calcifications and bowel, there is not much of a difference between AP and PA projections supporting the fact that all image quality is better in PA than AP according to three radiologists. The average percentage of effective skin dose along with different BMI categories was from 30 to 45 . In healthy patients, the average skin dose reduced around 32, while in overweight and obese patients it was 45 and 35 respectively.

In healthy BMI patients who already have small abdominal diameter thickness, there is no large area to be compressed in prone position which will result in no change or decrease in the effective skin dose. Similar results of dose reduction in patient entrance surface was observed in the research conducted by P.C. Brennan and E. Madigan (Brennan \&t Madigan, 2000), where an average of $38.6 \%$ reduction in lumbar spine imaging was reported when the patient was moved from AP to PA position for lumbar spine projection. Similar results in DAP and effective dose reduction by $27 \%$ and $53 \%$ respectively were found in the research conducted by Erna Alukic et al (Alukic et al., 2018), PA projection was used instead of AP projection in lumbar spine radiography (Frank et al., 1983). Another research called dose reduction in Lumbar spine radiography conducted by Heriard JB et al, mentioned that PA lumbar projection produced the benefit of reduced dose $(200+\%)$ to the eyes, ovaries, uterus and testis (Jb et al., 1993).

\section{CONCLUSION}

The practical implication of this technique does not require additional equipment and has no extra cost. In changing the technique from $\mathrm{AP}$ to $\mathrm{PA}$, the patient lies on his/her abdomen and achieved easily without affecting the time of the exam or disturbing the patient comfort. The results of our study, in line with previous studies, confirm that patient diameter strongly affects patient dosage. Finally, although AP abdomen is more preferable than PA among all radiology departments across the globe due to AP position is more comfortable for patients in all condition and sizes. Moreover, it is more comfortable for the technologist to face patient and look to his face expressions for better communication and assessment, PA projection justified to be simple method pertinent to dose reduction without any loss of diagnosis and image quality for the patient and no additional cost for the department. Its supporting and achieving strict adherence to one of the main principles of radiation protection policies, "as low as reasonably achievable" (ALARA).

\section{ACKNOWLEDGEMENTS}

We would like to thank and appreciate the whole radiology team of the King Abdulaziz Medical City, Riyadh, Saudi Arabia for their contribution to this study, done efficiently, and the practical aspect a success. Special thanks to the Radiologists who contributed, answered and reviewed the cases: Mohammed Faheem and Dr. Ahmed Zaidan.

Competing Interests: Authors have declared that no competing interests exist

\section{REFERENCES}

Alukic, E., Skrk, D., \&t Mekis, N. (2018). Comparison of anteroposterior and posteroanterior projection in lumbar spine radiography. Radiology and oncology, 52(4), 468-474. https://doi.org/10.2478/raon-20180021

Brennan, P. C; \& Madigan, E. (2000). Lumbar spine radiology: analysis of the poster anterior projection. European Radiology, 10(7), 1197-1201

Doktor, Klaus et al. "European guidelines on quality criteria for diagnostic radiographic images of the lumbar spine - an intra- and inter-observer reproducibility study." Chiropractic \&t manual therapies vol. 27 20. 1 May. 2019, doi:10.1186/s12998-019-0241-3

Don, S., MacDougall, R., Strauss, K., Moore, Q. T., Goske, M. J., Cohen, M., Lehman, L. (2013). Image Gently campaign back to basics initiative: Ten steps to help manage radiation dose in pediatric digital radiography. American Journal of Roentgenology, 200(5), W431W436. doi:10.2214/AJR.12.9895

Furlow, B. (2011). Radiation protection in pediatric imaging. Radiologic Technology, 82(5), 421-439. Retrieved from: http://www.radiologictechnology.org/ content/82/5/421.full.pdf + html?sid=6ecb0252-a24947f4-a2e9-a2270d95e125

Frank ED, Stears JG, Gray JE, Winkler NT, Hoffman AD. Use of the posteroanterior projection: a method of reducing $x$-ray exposure to specific radiosensitive organs. Radiol Technol. 1983; 54(5):343-347.

Ghearr, F. and Brennan, P. C. (1998). The PA projection of the abdomen: a dose reducing technique. International Journal of Diagnostic Imaging and Radiation Therapy, 4, 195-203.

Heriard JB, Terry JA, Arnold AL. Achieving dose reduction in lumbar spine radiography [published correction appears in Radiol Technol 1994 Mar-Apr; 65(4):219]. Radiol Technol. 1993; 65(2):97-106.

Marshall, N. W; Faulkner, K; Busch, P; Marsh, D. M; Ct Penning, H. (1994). A comparison of radiation dose in examination of the abdomen using different radiological imaging techniques. The British Journal of Radiology, 67, 478-484

Moore, Q. T. (2015). Manipulation of Projection Approach in Pediatric Radiography. Radiologic Technology, 86(5), 481-489.

Retrieved from: http://www.radiologictechnology.org/ content/86/5/481.full.pdf + html? sid=3c09da8b-3b7b4e8e-8cb5-d95e278b9bb8

The Ministry of National Guard Health Affairs' Administrative Policy and Procedures-APP 1430-43 (2017). Personal dosimetry, Riyadh, Saudi Arabia. 